\title{
Basal lamina and extracellular matrix alterations in the caudal neural tube of the delayed Splotch embryo
}

\author{
K.S. O'Shea and L.-H. J. Liu \\ Department of Anatomy and Cell Biology, University of Michigan Medical School, Ann Arbor, MI 48109 (U.S.A.)
}

(Accepted 14 April 1987)

Key words: Collagen type IV; Fibronectin; Heparan sulfate proteoglycan; Laminin; Mouse embryo; Neurulation

\begin{abstract}
Regional patterns of deposition of laminin (LN), fibronectin (FN), type IV collagen (IV), and heparan sulfate proteoglycan (HSPG) were examined during the formation of the caudal neural tube in embryos homozygous for the delayed Splotch gene and in their normal littermates. Delayed Splotch embryos had neural tube closure defects which extended from the posterior neuropore into the region formed by secondary neurulation. During posterior neuropore closure these components were normally restricted to forming basal laminae, with FN and HSPG additionally deposited in the mesenchyme. Unlike control embryos in which medial regions of the neuroepithelial basal lamina contained greatest amounts of all four, the dorsolateral zone contained less LN and IV and more FN and HSPG, in affected embryos these components were less densely deposited medially, reflecting perhaps the poor structural organization of the notochord. The neuroepithelial basal lamina was often disorganized and wavy compared to the linear pattern typical of controls. By the 12th day, the posterior neuropore of controls had closed and secondary neurulation was underway; however in delayed Splotch embryos, the neural folds remained widely splayed and epithelium newly formed via secondary neurulation extended that abnormally open configuration to the tip of the tailbud. In controls, with mesenchymal cell aggregation FN and HSPG were displaced from between cells to the forming basal lamina. As a central lumen formed within the aggregate LN and IV were added to the basal lamina, and the newly formed epithelium merged with the anterior neural tube. In delayed Splotch embryos, FN and HSPG were incompletely removed from aggregating cell surfaces, the normal morphogenetic cell shaping changes failed to occur and in many embryos a central lumen did not form; the overgrown, aggregated cells merging with the abnormally splayed anterior neural folds. In addition, the critical enrichment of FN and HSPG present between newly formed and consolidated neuroepithelium was displaced in delayed Splotch embryos.
\end{abstract}

\section{INTRODUCTION}

Unlike the cephalic neural folds which elevate, meet and fuse in the dorsal midline of the embryo to form the primitive $\mathrm{CNS}^{14}$, formation of the caudal portion of the neuraxis is complicated by a second series of events in which mesenchymal cells aggregate, form a central lumen, and merge with the neuroepithelium of the posterior neuropore region ${ }^{24}$. Like other mesenchymal/epithelial transitions, formation of the initial aggregate is accompanied by a loss of extracellular space; extracellular matrix components fibronectin and heparan sulfate proteoglycan are lost from between mesenchymal cells and appear in the forming basal lamina. With the appearance of a lu- men within the aggregate, the more structural components laminin and type IV collagen are also added to the basal lamina (BL) and the newly formed epithelium merges with the anterior neuroepithelium, extending the length of the CNS.

In embryos homozygous for the delayed Splotch gene $(S p d / S p d)$, the pattern of events involved in the formation of the caudal neural tube is strikingly abnormal; rather than extending its length, in delayed Splotch embryos the newly formed secondary epithelium merges with the abnormally splayed neural folds of the posterior neuropore extending the open neural tube. To determine the basis of these abnormal cellcell interactions, the pattern of deposition of extracellular matrix (ECM) and $\mathrm{BL}$ components fibronec-

Correspondence: K.S. O'Shea, Department of Anatomy and Cell Biology, University of Michigan Medical School, Ann Arbor, MI 48109, U.S.A. 
tin (FN), heparan sulfate proteoglycan (HSPG), laminin (LN) and type IV collagen (IV) were examined during posterior neuropore closure and secondary neurulation.

\section{MATERIALS AND METHODS}

Mice heterozygous for the delayed Splotch gene $(S p d /+)$ were initially obtained from Dr. H. Kalter, University of Cincinnati, and have been maintained in our colony for an additional 8 generations. Males and females were caged together overnight, the morning of finding a vaginal plug considered the first day of gestation.

On the afternoon of the 10th or 12th day, females were killed by cervical dislocation, uteri removed and embryos dissected from the chorion but not the amnion. Embryos were staged and separated into groups corresponding to: (1) embryos with lumbosacral neural tube defects; (2) developmentally delayed but otherwise normal embryos; and (3) normal embryos. All were immediately frozen in OCT embedding compound (Miles Labs.) in hexane cooled over an acetone-dry-ice slurry. Blocks were stored at $-90^{\circ} \mathrm{C}$ prior to sectioning.

Sagittal and transverse sections $(8 \mu \mathrm{m})$ through the lumbosacral neural folds and secondary neuroepithelium were cut using an International cryostat and collected on high molecular weight (g.t. 300,000) polyL-lysine-coated slides. For immunocytochemistry, sections were air-dried, exposed in a moist chamber to $5 \%$ normal goat serum followed by the primary antibody for $2 \mathrm{~h}$ at room temperature. They were then washed in PBS and exposed to the second antibody (goat anti-rabbit $\mathrm{IgG}$ ) conjugated to FITC (1:50, Cappel) for $30 \mathrm{~min}$ at room temperature. Sections were washed extensively in phosphate-buffered saline (PBS), coverslipped with glycerol containing $0.1 \%$ phenylenediamine and sealed with Kronig wax or nail polish. Controls were incubated in pre-immune serum or in PBS in place of the primary antibody.

Because of the extensive co-distribution and numerous interactions of these components ${ }^{22}$, a number of enzyme digestions were applied in an attempt to augment the staining or change the pattern of localization ${ }^{10}$. Collagenase $(275 \mathrm{U} / \mathrm{ml}, \mathrm{pH}=7.4$; Gibco $)$, Streptomyces hyaluronidase (100 TRU/ml; Miles), testicular hyaluronidase (400 units/ml; Cappel) were applied for $1 \mathrm{~h}$ at $37^{\circ} \mathrm{C}$ prior to exposure to the primary antibody. Exposure to testicular hyaluronidase significantly improved the intensity of fibronectin staining and was employed throughout these studies. No other treatment was effective and none altered the pattern of localization.

\section{Antibodies}

Antibodies to laminin were obtained from Dr. J.P. McCoy, Department of Pathology, University of Michigan; antibodies to type IV collagen from Dr. $\mathrm{H}$. Furthmayr, Department of Pathology, Yale University; and antibodies to heparan sulfate proteoglycan from Dr. J. Hassell, National Institute of Dental Research. All 3 antigens were isolated from EHS sarcoma. Antibodies to human serum fibronectin were purchased from Cappel laboratories. All were raised in rabbits, were affinity purified and exhibited no

Fig. 1. Lateral SEM and corresponding sagittal immunocytochemical preparations of control (A, C, E, G) and delayed Splotch (B, D, $\mathrm{F}, \mathrm{H}$ ) embryos. A: lateral view of the posterior neuropore region in a 10 th day control embryo. Arrows indicate the sides of the unfused neural folds. B: similar view of a 10th day delayed Splotch embryo illustrating the widely open neural folds of the posterior neuropore (arrowed). Note also the abnormal dorsal flexion of the region. C: by the 12th day, the posterior neuropore has completely closed and the tailbud lengthened considerably. D: tailbud region of a 12th day delayed Splotch embryo illustrating the open defect (arrowed) which extends to the tip of the tail. There is also an abnormal flexion of the region. E: sagittal section through the posterior neuropore region of a 10th day control embryo illustrating anti-HSPG staining of basal laminae of neuroepithelium (NE), notochord (N), hindgut (G). There is also mesenchymal staining and deposits of HSPG associated with the caudal extent of the neuroepithelium (arrowed). F: sagittal section through the posterior neuropore of a delayed Splotch embryo on the 10th day. Anti-HSPG immunoreactivity is associated with BL and is present in the mesenchyme. Rather than the more linear neuroepithelial BL profile seen in controls, HSPG staining is somewhat punctate and follows an irregular course (arrowed). Deposition at the crucial caudal merging zone is also absent. G: anti-FN staining of the tailbud of a control embryo on the 12th day. As at earlier stages, BL stain intensely, there is mesenchymal stain ing and taining of the tailbud of a control embryo on the 12th day. As at earlier stages, BL stain intensely, there is mesenchymal staining and deposition of FN in the zone of merging between consolidated and newly formed epithelia (arrowed). NE, neuroepithelium; $G$, hindgut. H: sagittal section through the tailbud of a 12th day delayed Splotch embryo in which the neural folds are widely splayed In addition to deposition in the BL of neuroepithelium (NE), notochord, (N) and hindgut $(\mathrm{G})$, there is FN staining at the base of the everted neuroepithelium (arrowed). Scale bars, $100 \mu \mathrm{m}$. 
cross-reactivity by ELISA. Anti-laminin was used at a concentration of 1:50, anti-fibronectin 1:10, anticollagen type IV $1: 100$, and anti-heparan sulfate pro-


teoglycan 1:20.

After staining, slides were viewed and photographed in a Leitz Dialux Orthoplan photomicros-
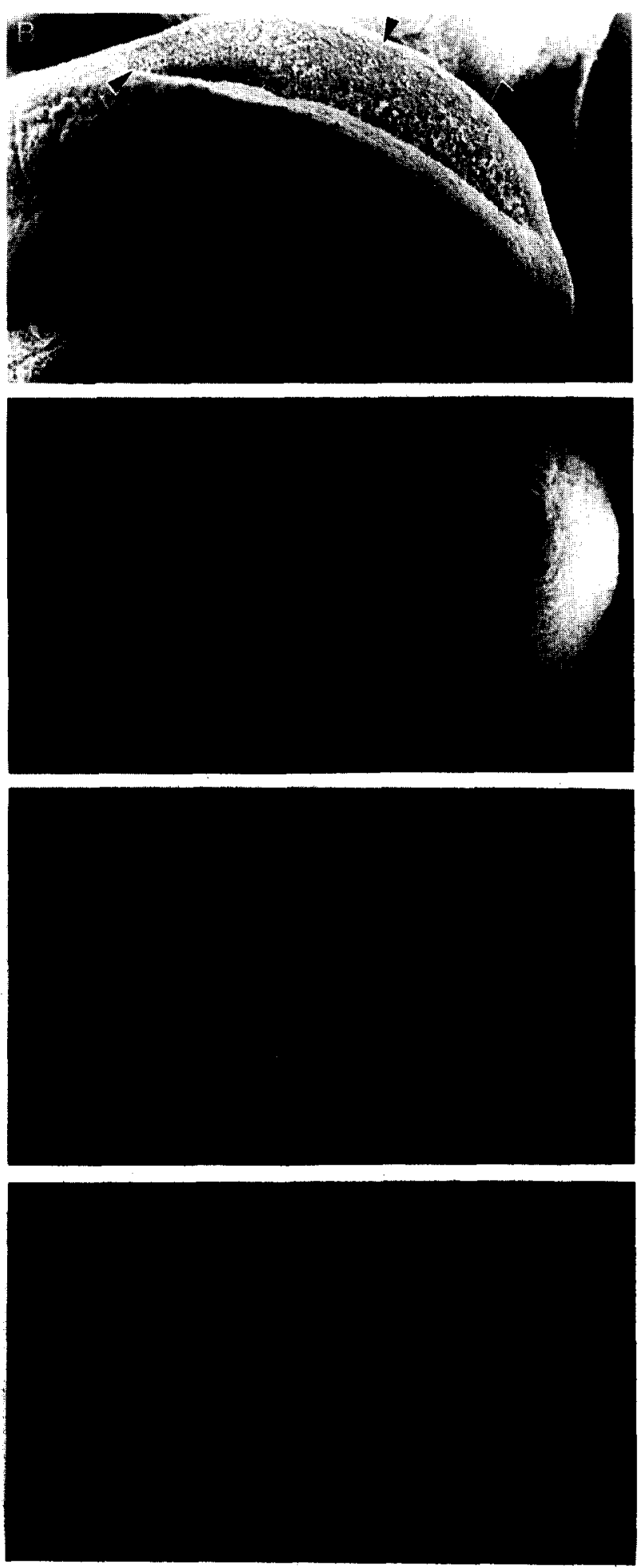
cope using UV light. Immunofluorescence was photographed using Kodak 2475 Recording film.

\section{RESULTS}

On the 10th day, the caudal neural folds were typically fused except at the posterior neuropore, where the neuroepithelium extended to the tip of the embryo as a trough-shaped thickening (Fig. 1A). A bulbous end region, the regressing primitive streak, was visible at its most caudal extent. In delayed Splotch embryos, rather than closing in the dorsal midline, the sides of the neural folds had failed to fuse and splayed laterally for a considerable distance (Fig. 1B). Rather than its more typical horizontal position, this region often appeared to have an abnormal dorsal flexion.

By the 12th day, the posterior neuropore had closed and the tailbud region had normally elongated considerably (Fig. 1C). In affected embryos, the neural tube remained unfused from the lumbar region to the tip of the tailbud (Fig. 1D). In addition, there was typically an abnormal flexure of this region which ranged in severity from a slight bend (Fig. 1D) to a kink so severe that dorsal regions of the open neural tube became fused.

Other than developmental delay, there were no differences observed between the small but apparently normal embryos (group 2) and the normal embryos (group 3). However, only embryos from the latter group were employed in the current investigation. More detailed reports of the nature and charac- teristics of the neural tube defects found in the allelic Splotch mutant are available elsewhere ${ }^{6,13,23}$.

In sagittal sections of control embryos, antibodies to laminin and type IV collagen were localized to basal laminae of the neuroepithelium, notochord, hindgut and developing blood vessels in the region. In addition, FN and HSPG (Fig. 1E) were found in the mesenchyme and both were densely deposited at the caudal extent of the neuroepithelium. In embryos in which posterior neuropore closure was delayed and the neural folds laterally splayed, the pattern of staining for $\mathrm{LN}$ and for IV was similar to that seen in control embryos with several important differences. First, the basal surface of the neuroepithelium, rather than its smooth, attenuated normal appearance, was often wavy and irregular. Secondly, staining around the notochord was often patchy and incomplete, reflecting its poor histological organization. The pattern of FN and HSPG (Fig. 1F) immunoreactivity was also abnormal, reflecting the structural alterations described above. In addition, they were notably absent from the most caudal region of the neuroepithelium, between consolidated neural tube and primitive streak (Fig. 1F), although the mesenchyme contained considerable FN and HSPG.

By the 12th day, LN and IV were present in basal laminae of neuroepithelium, notochord, and hindgut of control embryos. In addition, as on the 10th day, FN (Fig. 1G) and HSPG were found in mesenchyme and at the caudal tip of the neuroepithelium; the region where newly formed epithelium merges with the consolidated neuroepithelium. In delayed Splotch

Fig. 2. Transverse sections from the caudal tip of the embryo cephalad in control (A-D) and delayed Splotch (E-H) embryos on the 10 th day. A: section through the regressing primitive streak (PS) in a control embryo illustrating very slight deposition of IV along its base. B: cephalad, the neural folds elevate and approach in the dorsal midline. Anti-type IV collagen staining is associated with BL of the neuroepithelium (NE), notochord (N), hindgut and of blood vessels. Its deposition is most dense near the midline, less dense toward the dorsolateral margins of the neural folds in the region of eminent neural crest cell migration. $\mathrm{C}$ : section through the primitive streak illustrating anti-FN immunoreactivity in the region. There is scattered mesenchymal staining and slight immunoreactivity associated with the base of the streak and with BL of forming blood vessels. D: section through the posterior neuropore illustrating anti-FN staining associated with basal laminae of notochord (N), neuroepithelium (NE), and hindgut. Note the lateral movement of the BL by the neural crest (arrowed). E: section through the anterior primitive streak illustrating anti-LN immunoreactivity associated with forming blood vessels within the mesenchyme and slight staining of the base of the thinned streak. F: section through the posterior neuropore. The neural folds have failed to elevate and are present on the dorsal surface of the embryo as a flat plate. Anti-LN staining is associated with the neuroepithelial (NE) basal lamina and BL of the hindgut. There is no immunoreactivity in the neural crest migratory pathway (arrowed). In the absence of a notochord the most intensely staining zone of the neuroepithelial basal lamina is found at its lateral margin. G: primitive streak from a delayed Splotch embryo illustrating anti-FN immunoreactivity in the mesenchyme and particularly densely deposited at the base of the regiessing streak (arrowed). $\mathrm{H}$ : more cephalic region through the posterior neuropore illustrating the failure of neural fold elevation and little organized deposition of $\mathrm{FN}$ at the base of the neuroepithelium (NE), although it is present within the mesenchyme. Scale bars, $100 \mu \mathrm{m}$ 
embryos, the neural folds were unelevated and unfused, remaining plate-like on the surface of the embryo. BL of neuroepithelium, hindgut and of the no- tochord contained all four components. In these embryos, the neuroepithelium often extended sheet-like over the caudal tip of the embryo, with FN particular-
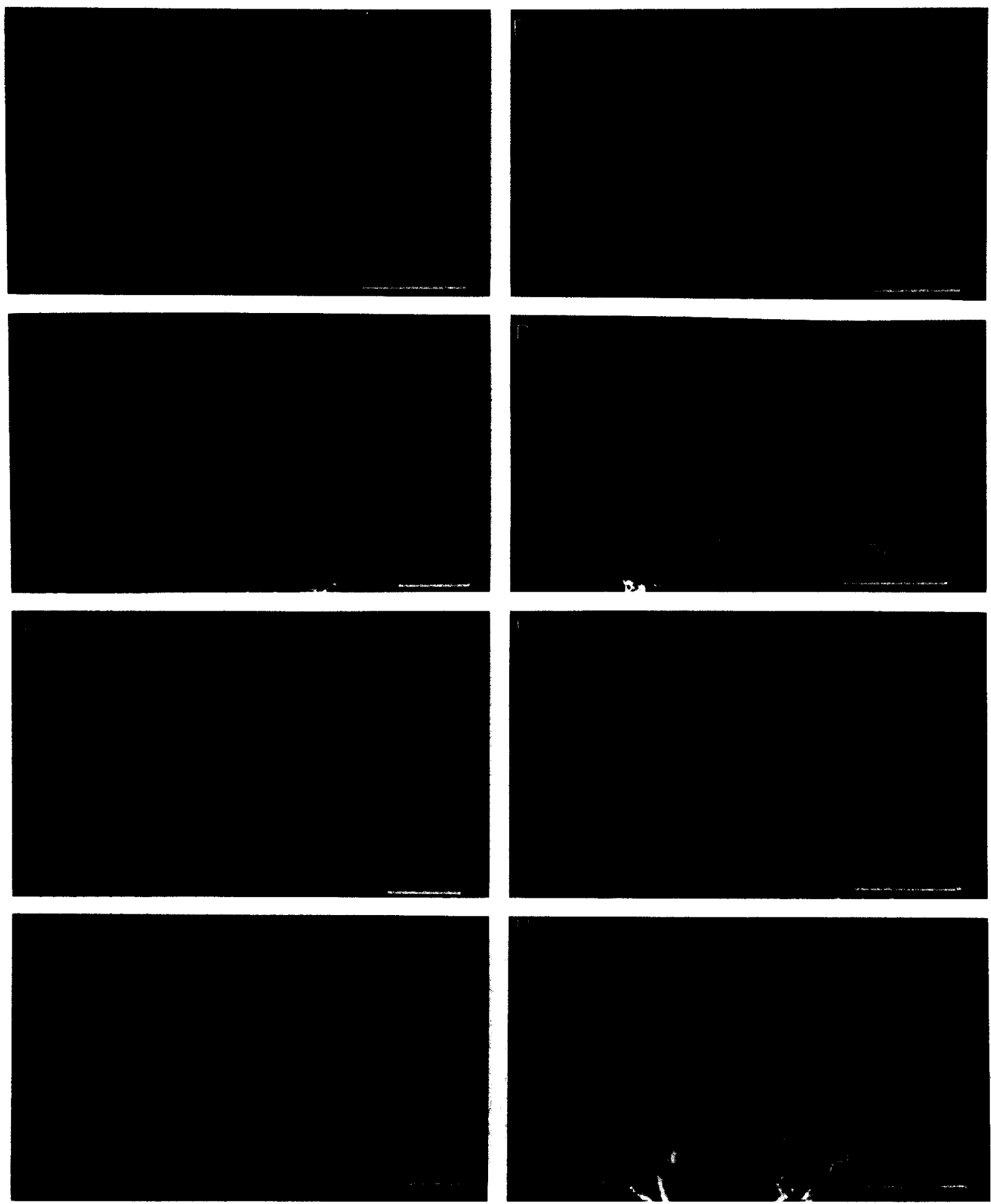
ly deposited at its base (Fig. 1H).

When examined in cross-sections, BL components were present at the base of the primitive streak - LN and IV (Fig. 2A) were less prominent in this region, while HSPG and FN (Fig. 2C) were more densely deposited here. Cephalad, the neural folds elevated and the notochord became organized at the midline. Hindgut, notochord and neuroepithelial basal laminae stained intensely for LN, FN, HSPG and IV (Fig. 2B). HSPG and FN (Fig. 2D) were also mesenchymal components. All 4 were most densely deposited in the neuroepithelial basal lamina in the medial region, staning slightly less intensely in the lateral zone, and in regions of active neural crest cell migration at the dorsolateral boundary, structural BL components were less densely deposited (Fig. 2B) while there was increased deposition of FN (Fig. 2D) and of HSPG.

In embryos with neural tube closure defects, the pattern was very different. The base of the primitive streak and blood vessel BL stained as in controls for IV and LN (Fig. 2E), but the base of the thinned streak was particularly enriched in FN (Fig. 2G). Cephalad, the deposition of structural BL components was similar to that seen in controls, LN and IV (Fig. $2 \mathrm{~F}$ ) were found in $\mathrm{BL}$ of the neuroepithelium and hindgut, but there was often no evidence of an organized notochord in these embryos. Like the primitive streak, the neuroepithelium was also very reduced in thickness. The pattern of immunoreactivity in the neuroepithelial $\mathrm{BL}$ was also altered; the most intensely staining region was in the lateral margins, there was little staining medially or in the neural crest re- gion (Fig. 2F). There was considerable mesenchymal stain for FN and for HSPG, although their deposition in the neuroepithelial basal lamina was somewhat patchy and non-linear (Fig. $2 \mathrm{H}$ ).

During secondary neurulation the pattern of cellular changes ${ }^{24}$ and immunocytochemical localization of $\mathrm{ECM}$ and $\mathrm{BL}$ components ${ }^{21}$ did not differ from previous reports in control embryos. Briefly, at the caudal tip of the tailbud, unorganized mesenchymal cells were surrounded by FN and HSPG. At this level LN and IV were restricted to BL of forming blood vessels. Cephalad, mesenchymal cells formed a dorsal aggregate with unorganized deposits of IV (Fig. $3 \mathrm{~A})$ and LN only rarely observed at its base. At this level, FN and HSPG (Fig. 3C) were lost from between aggregated cells and were deposited at their base.

As a lumen began to form within the aggregated cells, LN and IV (Fig. 3B) were also densely deposited in the forming BL. FN and HSPG (Fig. 3D) clearly separated the base of cavitating cells from the surrounding mesenchyme and both were general mesenchymal components.

In delayed Splotch embryos, the pattern of events was considerably different. HSPG and FN were found in the unorganized mesenchyme of the tailbud. Cephalad, rather than forming a discrete aggregate near the dorsal surface, aggregated cells composed a considerable proportion of the region; located in dorsal, dorsomedial zones and forming columns along the lateral surface of the embryo (Fig. 3E). Two patterns of events followed. In the first, the central portion of the aggregate either failed to canalize or

Fig. 3. Transverse sections through the tailbud region of control (A-D) and delayed Splotch (E-H) embryos on the 12th day illustrating the pattern of deposition of $\mathrm{BL}$ and $\mathrm{ECM}$ components during mesenchymal aggregation $(\mathrm{A}, \mathrm{C}, \mathrm{E}, \mathrm{G})$ and cavitation $(\mathrm{B}, \mathrm{D}, \mathrm{F}, \mathrm{H})$. A: caudal tip of the tailbud in a control embryo illustrating the very scattered anti-IV staining. There is very little IV associated with the mesenchymal aggregate, although occasional punctate staining is seen at its base (arrowed). B: more cephalic section through the region of cavitation of the aggregate, illustrating the dense deposition of IV in the forming basal lamina of the epithelium (arrowed) and in BL of blood vessels. C: section through the aggregation zone of an additional control embryo illustrating the deposition of HSPG at the base of the aggregated cells (arrowed). HSPG was also present in the mesenchyme and in blood vessel basal laminae. D: cephalad, the aggregate begins to canalize and HSPG forms a linear deposit at the base of the epithelium (arrowed). It is also associated with blood vessel basal laminae and is a general mesenchymal component. E: anti-IV staining in the aggregation zone in a de layed Splotch embryo. Note the considerable increase in the number of cells in this region compared to control embryos. There is no organized deposition of IV at the base of the aggregate. F: cephalad, at the level where cavitation would normally occur, type IV collagen outlines the base of the cells (arrowed), and is found in the mesenchyme. G: section through the aggregation zone in a delayed Splotch embryo. An increase in the number of aggregating cells is seen in this embryo.However, FN remains on the surface of some of them and is less linearly displaced to their lower borders (arrowed). FN is also present in blood vessel BL and in the mesenchyme. H: section through the region where cavitation would normally occur illustrating the immense overgrowth of the region and the deposition of FN between and at the basal surface (arrowed) of the epithelial cells. Scale bars, $100 \mu \mathrm{m}$. 
formed a very small lumen, which then merged with the abnormally open cephalic folds (Fig. 3F). In the second situation, cells failed to undergo the typical pattern of morphogenetic cell-shaping change and remained abnormally flattened on the surface of the embryo.
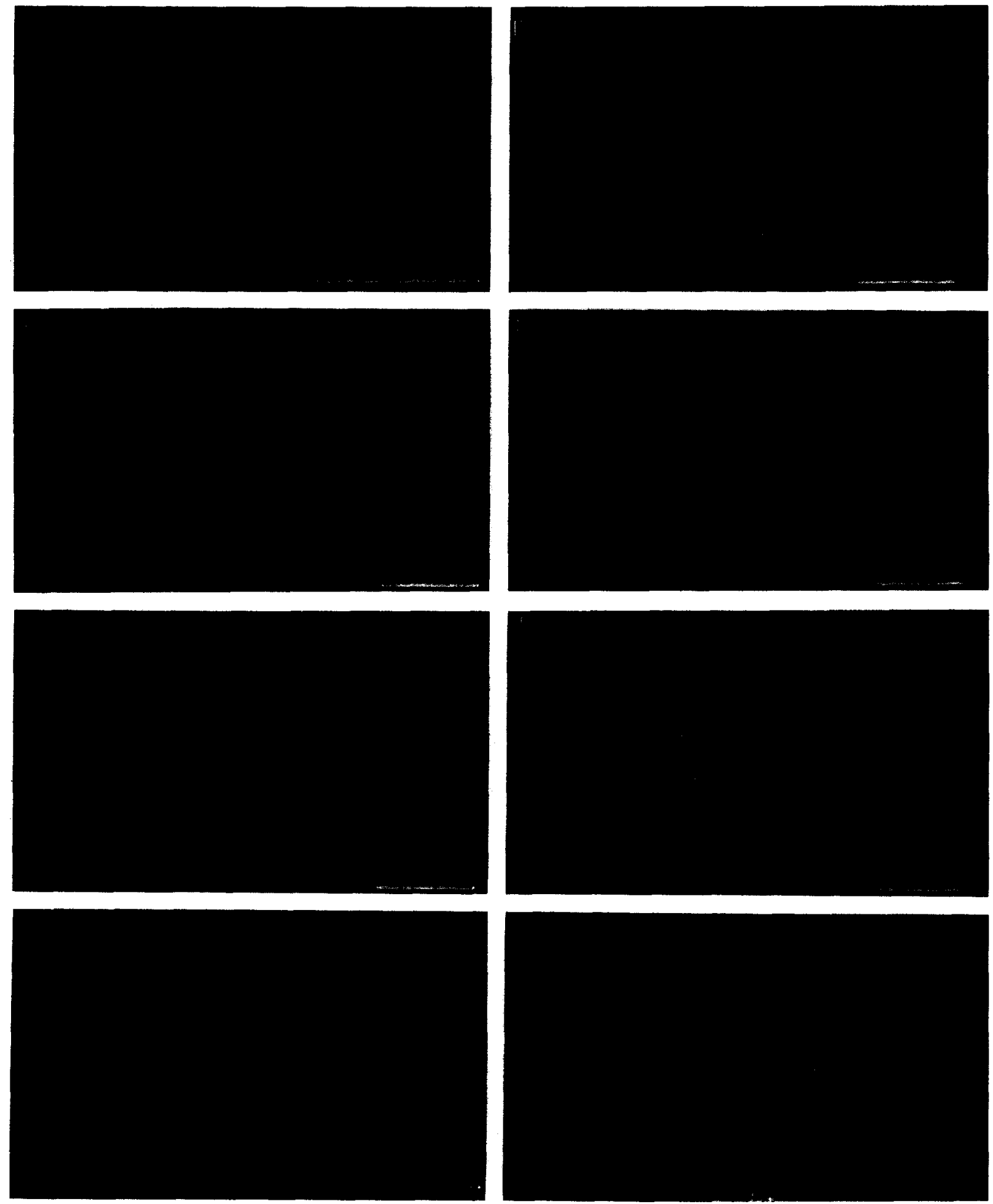
In the first situation, LN and IV (Fig. 3E) were not seen at the base of the initial aggregate, but were found at the base of the cells as they merged with the open neural folds (Fig. 3F). HSPG and FN (Fig. 3G, $\mathrm{H}$ ) were present at the base of the aggregate and with abortive cavitation; however, rather than being excluded from between aggregated cells, FN particularly remained between them. This occurred both at the level of initial aggregation (Fig. 3G) and in the zone where cavitation of the aggregate would normally occur (Fig. 3H).

In the second situation, the aggregate failed to form and the neuroepithelium extended from the posterior neuropore to the tip of the embryo as a flat sheet of cells. The pattern of immunoreactivity and histological appearance of the neuroepithelium in these embryos did not differ from that seen in other delayed Splotch embryos on the 10th day.

\section{DISCUSSION}

Embryonic basal laminae are thought to both support epithelial structures and to play an important role in information transfer between epithelium and subjacent mesenchyme ${ }^{1,9}$. In the current investigation neuroepithelial BL abnormalities and alterations of the mesenchymal extracellular matrix were observed in delayed Splotch embryos. During posterior neuropore closure, the normal pattern of deposition in the neuroepithelial basal lamina was altered, with the typically most densely staining midline region reduced significantly in all 4 components. The lack of midline BL staining in these embryos likely reflects the abnormal organization of the notochord and lack of interaction between neuroepithelium and notochord. In addition, rather than its typical linear appearance, the neuroepithelial BL of these embryos was often wavy and disorganized. These observations provide some support for the suggestion that the neuroepithelial BL functions as a solid substratum against which morphogenetic cell shaping alterations required for neurulation can occur, as the delayed Splotch neuroepithelium was very thinned compared with controls.

Anomalies of the neuroepithelial BL produced by exposure of neurulating embryos to agents which alter the synthesis or deposition of BL and ECM components, e.g., $\beta$-D-xyloside ${ }^{16}$, cis-hydroxyproline ${ }^{19}$, or to tunicamycin ${ }^{18}$, also produce neural tube defects. Similarly, a number of neurological mutants of the mouse exhibit both neural tube closure defects and basal lamina/extracellular matrix abnormalities, loop-tail ${ }^{4,29-31}$, Splotch $^{15}$, Patch $^{7}$, the $T$ mutants ${ }^{11,28}$, trisomy for chromosomes 12 , and 14 (ref. 17), Snell's translocation $(\mathrm{T}(1,2) 1 \mathrm{Sn})^{20}$. However, the underlying basis for this association and its source whether mesenchyme and/or epithelium is abnormal - remain to be determined.

The precise role of the neuroepithelial BL in CNS histogenesis has received relatively little attention. In addition to providing a solid substrate for cell orientation and integrated cellular movements (such as neurulation), microheterogeneities in composition of the $\mathrm{BL}$ might play a significant role in producing regional patterns of proliferation within the nervous system (e.g., neuromere formation), may underlie the escape of cells and cell processes from the CNS (e.g. neural crest and growth cones), and may play a role in cell migratory processes along basal lamina.

Notochordal anomalies and abnormalities of axial elongation co-occur with neural tube defects following experimental manipulation ${ }^{12}$, and in neurological mutants: loop-tail ${ }^{27}$, curly tail ${ }^{3}, T, t 9$ (ref. 28), but have not previously been reported in delayed Splotch or Splotch mutants. In the delayed Splotch embryo, presence of a notochordal anomaly is consistent with the observed flexion anomalies of the posterior neuropore region and of the tailbud. Flexion anomalies of this type have previously been suggested to play a primary role in the genesis of neural tube defects ${ }^{3}$ and may reflect the degree of delay in neural tube closure typical of embryos with varying doses of the mutant gene $\mathrm{g}^{6,13}$. Because notochordal cells (like secondary neuroepithelial cells) must first aggregate within the mesenchyme and then organize, an anomaly of the mesenchymal cells themselves or of their ECM might similarly produce the observed notochordal anomalies.

During secondary neurulation, the pattern of deposition of ECM but not of BL components was abnormal. FN and HSPG remained abnormally present on some of the cells in the dense aggregate rather than being displaced into the forming BL. Interestingly, these cells were somewhat abnormally shaped and often failed to interact with neighboring cells to form a central lumen. It would be of considerable in- 
terest in this regard to determine the extent of junction formation between them.

Since a considerable proportion of the tailbud appears to be of neural crest origin ${ }^{25}$, and because Splotch embryos are characterized by anomalies of neural crest cell migration ${ }^{23}$, failure of these cells to migrate normally from the epithelium or to interact with the ECM might produce the secondary neuroepithelial abnormalities observed in the current investigation.

Because both HSPG and FN appear to have transmembrane connections with intracellular actin ${ }^{26,32}$, the abnormal persistence of these two components on the surface of aggregated cells could influence their shape, migration, and amount of extracellular space in the region. Thus, initiation of the epithelialization cascade would be inhibited, producing the ectopic aggregate of cells observed in these embryos. Additional support for this suggestion comes from observations of abnormal FN retention on neural crest cell surfaces in Splotch embryos during dorsal root ganglia formation ( $O$ 'Shea, unpublished observations).

Interestingly, in the $t 9$ mutant, the caudal neural tube also forms abnormally. In this case a series of ectopic aggregates, rather than a single aggregate of cells forms in the region ${ }^{28}$. It would be of considerable interest to determine if there is an abnormality in the pattern, time of appearance, or relative pro-

\section{REFERENCES}

1 Bernfield, M., Banerjee, S.D., Koda, J.E. and Rapraeger, A.C., Remodelling of the basement membrane: morphogenesis and maturation. In Basement Membranes and Cell Movement, CIBA Foundation Symposium Vol. 108, Pitman, London, 1984, pp. 179-191.

2 Brown, P.J. and Juliano, R.L., Expression and function of a putative cell surface receptor for fibronectin in hamster and human cell lines, J. Cell Biol., 108 (1986) 1595-1603.

3 Copp, A.J., Relationship between timing of posterior neuropore closure and development of spinal neural tube defects in mutant (curly tail) and normal mouse embryos in culture, J. Embryol. Exp. Morphol., 88 (1985) 39-54.

4 Copp, S.N. and Wilson, D.B., Cranial glycosaminoglycans in early embryos of the Loop-tail $(L p)$ mutant mouse, $J$. Craniofac. Genet. Dev. Biol., 1 (1981) 253-260.

5 Costanzo, R., Watterson, R.L. and Schoenwolf, G.D., Evidence that secondary neurulation occurs autonomously in the chick embryo, J. Exp. Zool., 219 (1982) 233-240.

6 Dempsey, E.E. and Trasler, D.G., Early morphological abnormalities in Splotch mouse embryos and predisposition portions of ECM components, or in their cellular receptors in these embryos as well.

With continued development, secondary neuroepithelial cells did not form a continuous cord-like structure, but extended the abnormally open neural tube into the tail. Thus it appears that the newly formed secondary neuroepithelial cells align along and form permanent junctions with cells of the anterior neuroepithelium, using it as a template. This is contrary to the situation in the chick embryo, where if the posterior neuropore is prevented from closing, the secondary neuroepithelium forms an autonomous cord of cells ${ }^{5}$. The presence of HSPG and of FN in this region may play a crucial role in the merging of these two populations, as both have been shown to mediate cell-cell interactions, to promote cellular migrations, and $\mathrm{FN}$ to participate in the maintenance of extracellular spaces with the embryo ${ }^{8,33}$.

Current investigations are in progress to extend these studies to the ultrastructural level and to examine the distribution of antibodies to the fibronectin receptor $^{2}$ in these embryos.

\section{ACKNOWLEDGEMENTS}

Supported by NIH Grant NS-21108 and grants from the Spina Bifida Association of America and the Easter Seal Research Foundation.

to gene- and retinoic acid-induced neural tube defects, $T e$ ratology, 28 (1983) 461-472.

7 Erickson, C.A. and Weston, J.A., An SEM analysis of neural crest migration in the mouse. J. Embryol. Exp. Morphol., 74 (1983) 97-118.

8 Hascall, V.C. and Hascall, G.K., Proteoglycans. In Hay, E.D. (Ed.), Cell Biology of Extracellular Matrix, Plenum, New York, 1981, pp. 39-63.

9 Hay, E.D., Extracellular matrix, J. Cell Biol., 91 (1981) 205S-233S.

10 Holund, B. and Clemmensen, I., The value of hyaluronidase treatment of different tissues before demonstration of fibronectin by the indirect immunoperoxide technique, Histochemistry, 76 (1982) 517-525.

11 Jacobs-Cohen, R.J., Spiegelman, M. and Bennett, D., Abnormalities of cells and extracellular matrix of $T / T$ embryos, Differentiation, 25 (1983) 48-55.

12 Jacobson, A.G., Further evidence that formation of the neural tube requires elongation of the nervous system, $J$. Exp. Zool., 230 (1984) 23-28.

13 Kapron-Bras, C.M. and Trasler, D.G., Gene-teratogen interaction and its morphological basis in retinoic acid-in- 
duced mouse spina bifida, Teratology, 30 (1984) 143-150.

14 Karfunkel, P., The mechanism of neural tube formation, Int. Rev. Cytol., 181 (1972) 289-302.

15 Morris, G.L. and O'Shea, K.S., Anomalies of neuroepithelial cell associations in the Splotch mutant embryo, Dev. Brain Res., 9 (1983) 408-410.

16 Morriss-Kay, G.M. and Crutch, B., Culture of rat embryos with B-D-xyloside: evidence of a role for proteoglycans in neurulation. J. Anat., 134 (1982) 491-506.

17 Morriss-Kay, G. and Putz, B., Abnormal neural fold development in mouse trisomy 12 and trisomy 14. II. LM and TEM, Brain Res. Bull., 16 (1986) 825-832.

18 O'Shea, K.S., Tunicamycin-induced defects in the mouse embryo: an in vitro study. In S.R. Hawkes and J.L. Wang (Eds.), Extracellular Matrix, Academic, New York, 1982, pp. 147-151.

19 O'Shea, K.S., Type IV collagen in neurulation, Soc. Neurosci. Abstr., 12 (1986) 316.

20 O'Shea, K.S., Ultrastructural analysis of chromosome translocation-induced neural tube defects, Brain Res. Bull., 16 (1986) 833-844.

21 O'Shea, K.S., Differential deposition of basement membrane components during formation of the caudal neural tube in the mouse embryo, Development, 99 (1987) $509-519$.

22 Ruoslahti, E. and Engvall, E., Complexing of fibronectin, glycosaminoglycans and collagen, Biochim. Biophys. Acta, 631 (1980) 350-358.

23 Russell, W.L., Splotch: a new mutation in the house mouse, Genetics, 32 (1947) 102.

24 Schoenwolf, G.C., Histological and ultrastructural studies of secondary neurulation in mouse embryos. Am. J. Anat. 169 (1984) 361-376.

25 Schoenwolf, G.C., Chandler, N.B. and Smith, J.L., Analysis of the origins and early fate of neural crest cells in caudal regions of avian embryos, Dev. Biol., 110 (1985) 467-479.

26 Singer, I.I., The fibronexus: a transmembrane association of fibronectin containing fibers and bundles of $5 \mathrm{~nm}$ microfilaments in hamster and human fibroblasts, Cell, 16 (1979) 675-685.

27 Smith, L.J. and Stein, K.F., Axial elongation in the mouse and its retardation in homozygous looptail mice, J. Embryol. Exp. Morphol., 10 (1962) 73-87.

28 Spiegelman, M., Electron microscopy of cell associations in $T$-locus mutants. In K. Elliot and M. O'Connor (Eds.), Embryogenesis in Mammals, Elsevier, Amsterdam, 1976, pp. 199-226.

29 Wilson, D.B., Histochemical analysis of the neural basal lamina in the hindbrain region of exencephalic mutant mice, J. Craniofac. Genet. Dev. Biol., 5 (1985) 363-371.

30 Wilson, D.B., Ultrastructure of the neural basal lamina in loop-tail mice. Acta Anat., 123 (1985) 77-81.

31 Wilson, D.B., Ultrastructural analysis of basal neuroepithelial cells in dysraphic mice, Virchows Arch., 48 (1985) 9-17.

32 Woods, A., Hook, M., Kjellen, L., Smith, C.G. and Rees, D.A., Relationships of heparan sulfate proteoglycans to the cytoskeleton and extracellular matrix of cultured fibroblasts, J. Cell Biol., 99 (1984) 1743-1753.

33 Yamada, K.M., Cell surface interactions with extracellular matrix material, Annu. Rev. Biochem., 52 (1983) 761-799. 